\title{
Calibrating Multispectral Imaging System for Biological Particle Analysis under Transmitted Illumination
}

\author{
J. R. Thigpen, ${ }^{*}$ S. Shah, ${ }^{*}$ and F. A. Merchant**
}

* Quantitative Imaging Laboratory, University of Houston, Department of Computer Science, Houston, TX 77204-3010

** ADIR, LLC., 2450 South Shore Blvd., Suite 305, League City, TX 77573

Spectral imaging systems have long been used in the fields of astronomy and remote sensing. More recently, such systems have been coupled to microscopes for use in medical imaging applications such as karyotyping of hybridization experiments and general cell visualization [1-4]. Spectral imaging allows for the measurement of the wavelength spectrum (transmitted or emitted) at every pixel of a two-dimensional image, thereby, combining the features of spectroscopy and imaging to provide new insights into specimen structure and composition, allowing the spatial detection of subtle spectral changes [5]. In this paper, we describe a multispectral imaging system and its calibration for measuring the absorption of light at different wavelengths through biological particles. Biological particles can be comprised of several different particle types including red blood cells, red blood cell ghosts (red blood cells which have ruptured leaving only their membranes), white blood cells, bacteria, and several others. Characterization of these particles facilitates the diagnosis of various diseases. Spectral profiles of biological particles may increase the accuracy of classification during microscopic examination procedures which will enable better diagnoses.

In composite biological material, the interpretation of the spectra is a complicated problem. This is because a charge-coupled device (CCD) camera used for data acquisition does not have a uniform quantum efficiency across the excitation spectrum, leading to interference effects. In addition, energy variation of the emission spectra of the light source with respect to wavelength necessitates calibration of the imaging system in order for the true spectral signatures of the particles to be measured accurately. We use a grating-based spectral light source connected to the transmitted light port on a standard optical microscope which allows 2D image acquisition using a high resolution CCD camera. Images are collected from $400 \mathrm{~nm}$ to $700 \mathrm{~nm}$ in $10 \mathrm{~nm}$ increments, resulting in a stack of 31 images for each field of view. Our calibration approach involves normalizing the camera's exposure for each wavelength under the assumption that incident light should be uniform across all wavelengths in which we are interested and measured as half the dynamic range of the camera. As such, a least square error solution is used to compute the proper exposure value for each wavelength. Figure 1(a-b) and (c-d) show uncalibrated and calibrated images at 400nm and 500nm, respectively. The background variation is clearly evident in the uncalibrated images while after calibration the background intensity is equalized. This allows us to quantitate the true change in light transmission through particles of interest.

To compute the spectral profile of biological particles, we measure the amount of light transmitted through the specimen and divide by the amount of incident light (the light unobstructed by any specimen). This allows us to determine the amount of light absorbed by the specimen at each individual wavelength. Doing this across the visible spectrum creates a profile for that particle type. More formally, if we let $I_{t}$ be the transmitted light and $I_{i}$ be the incident light, then we can define a Transmission Factor $\mathrm{T}=\log \left(\mathrm{I}_{\mathrm{t}} / \mathrm{I}_{\mathrm{i}}\right)$, which we can plot as a function of wavelength. The curve of the transmission factor as a function of wavelength is known as the spectral profile. To evaluate our 
calibration procedure, we compare the computed spectral profile of WBC and RBC to the known profiles obtained by spectroscopy [6]. The computed and known profiles are shown in figures 2 and 3 , respectively. Both the computed and published profiles tend to peak around $540 \mathrm{~nm}$ and have a similar shape.

1. E. Kim et. al. A high-resolution multi-spectral imaging system for small satellites. Acta Astronautica, 52(9-12):813-818, 2003.

2. S. Tsunoda et. al. Novel spectral imaging system combined with confocal laser microscopy for fish analysis of interphase nuclei. Cytometry, page 139, 2002.

3. J. Kerekes and J. Baum. Spectral imaging system analytical model for subpixel object detection. IEEE Transactions on Geoscience and Remote Sensing, 40(5):1088-1101, 2002.

4. D. Maiti et al., Proton gradients in human breast cancer cells determined by confocal, multiphoton, and spectral imaging microscopy. FASEB Journal, 17(4):A467, 2003.

5. Y. Garini et al., Spectral Bio-Imaging. Fluorescence Imaging Spectroscopy and Microscopy, Chemical Analysis Series. John Wiley \& Sons, New York, 1996.

6. S. Kawata and K. Sasaki. Fluorescence Imaging Spectroscopy and Microscopy, chapter Multispectral Image Processing for Component Analysis. Chemical Analysis Series. John Wiley \& Sons, New York, 1996.

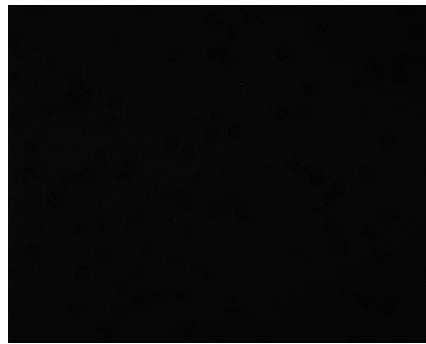

(a) 400nm @ 10ms exp.

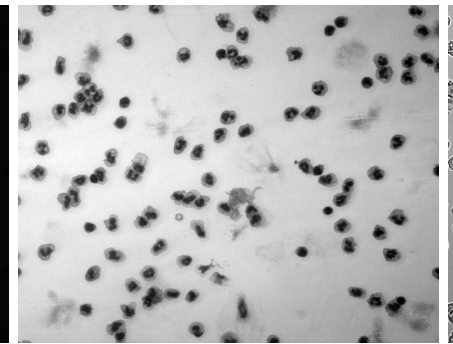

(b) 500nm@10ms exp. Figure 1. Uncalibrated and Calibrated Exposures.

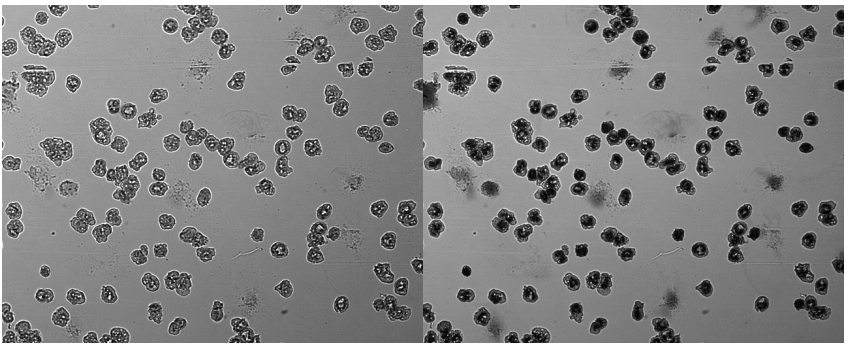

(c) 400nm @ 8000ms exp. (d) 500nm @ 180ms exp.

WBC - RBC Spectral Profile

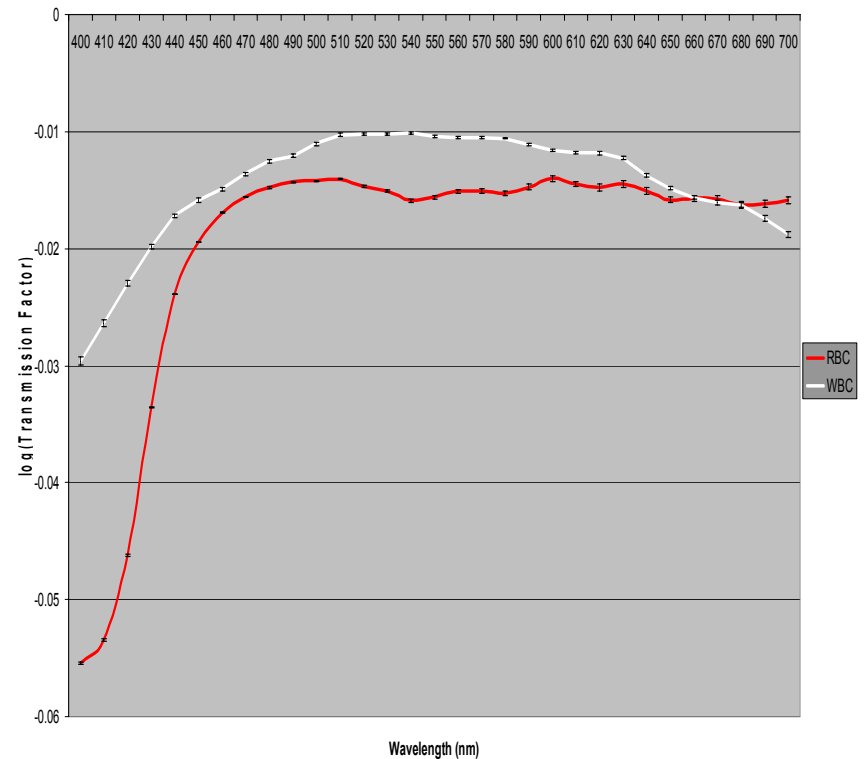

Figure 2. Calculated profiles of WBC and RBC after calibration.

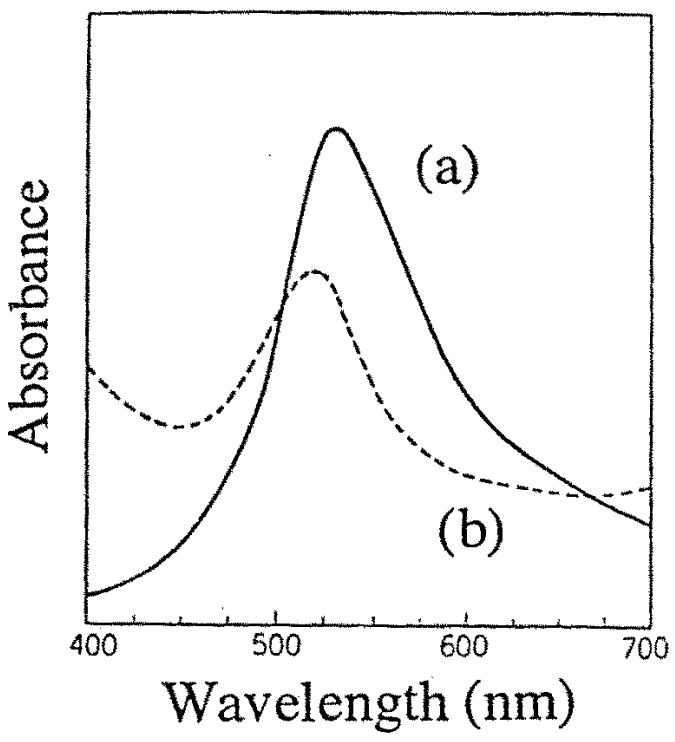

Figure 3. Published profiles of (a) WBC and (b) RBC from spectroscopy. 